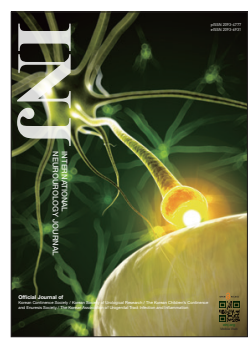

\title{
Editorial
}

Int Neurourol J 2019;23(Suppl 1):S1-2

https://doi.org/10.5213/inj.1920edi.001

pISSN 2093-4777 · eISSN 2093-6931

\section{How to Interpret the Effects Shown in Animal Study}

\author{
Khae Hawn Kim id https://orcid.org/0000-0002-7045-8004 \\ Department of Urology, Gachon University Gil Medical Center, Gachon University of School of Medicine, Incheon, Korea \\ E-mail: kimcho99@gilhospital.com
}

Some hospitals, oriental medicine clinics, and even the media often promote medicines and treatment methods on the basis of a few papers that present findings of efficacy from rat experiments. An example of such a statement would be, "A drug candidate with a much more powerful effect than existing drugs was identified." The unstated implication is that these effects were identified through the results of an animal experiment (most commonly a rat experiment). Another example is furnished by statements such as, "The effect of the drug was proven by articles published in an SCI (Science Citation Index) indexed journal, and a new treatment method was discovered, from which many patients will benefit." Again, left unstated is that the articles in question presented results from animal experiments.

However, the results of rat experiments should not be applied directly to humans, meaning that they should not be interpreted with excessive confidence. In order of frequency, the animals that are used in animal experiments in Korea are rodents, fish, birds, rabbits, and other mammals. Generally, novel drugs should be developed through a strict sequence of clinical trials. As part of this process, scientists evaluate the adverse effects or toxicity of a drug through rat experiments before performing an actual clinical trial. Since clinical trials impose costs of hundreds of billions to trillions of won and require a long time (sometimes more than 10 years), it is important to determine whether a substance is worth the investment of a clinical trial. Therefore, a clinical trial of a drug candidate can only be launched when the drug is confirmed not to have any problems in several stages of rat experiments. In other words, several stages of preclinical trials using animals play the role of filtering drug candidates through safety or toxicity tests. However, it cannot be expected that the positive effects of a drug shown in rat experiments will be the same in humans. According to the Food and Drug Administration, the chance that a drug candidate that went through preclinical trials and phase I clinical trials (conducted on a small number of people) will be sold on the market after going through phase II and III clinical trials is only $9.6 \%$. For this reason, it is generally accepted that one cannot say that "a drug is effective" or "superior to existing drugs" based on effects that have only been observed in rat experiments. Rats and humans belong to different orders within the class of mammals; as such, they are fundamentally different species with important genetic differences. As medical scientists, we interpret the effects observed in rat experiments as only applicable to rats until they are also observed in a clinical trial. In fact, there are many instances where drugs and illnesses have different effects between humans and rats. Examples include hantavirus, the bubonic plague, and rodent-borne hemorrhagic fevers, all of which do not cause any illness in rats but are potentially lethal diseases in humans. Drugs also show different effects in rats and humans because of the major differences between these species; in particular, drugs for brain diseases must be approached carefully.

Degenerative brain diseases that develop in humans, such as Parkinson disease, dementia, and depression are difficult to induce in animal models, particularly rats, and even when it is possible to do so, the mechanisms are not the same as in humans. This is why it is inappropriate to assume that a drug will have the same effects in rats and in humans. Moreover, a par- 
ticularly rigorous approach is needed in the field of neurourology, in which neurological disorders are associated with target organs [1]. Likewise, the effects of medicines, procedures, and operations (interventional and surgical treatments) should not be considered to have been scientifically proven because the results of related rat experiments have been published in international journal articles. Journal articles only indicate that a drug candidate is likely to have an effect from a scientific perspective, and many scientists then conduct further experiments to confirm that possibility. I think this is a very valuable virtuous circle of science. However, promoting the results of an animal experiment as indicative of efficacy in humans or exaggerating the findings of an international article based on rat experiments for use in advertisements could be considered false advertising, and such practices prevent the painstaking efforts of medical scientists from being evaluated fairly.

Let us suppose that a drug or treatment method is developed. Compounds or single substances are prepared and evaluated directly in a disease model (in vivo). Furthermore, as spe- cific proteins that cause certain diseases have been identified, in vitro analysis using proteins or cells has become a major research method. However, it may be the case that a certain substance or drug controls other proteins in addition to the initial target protein. By applying this insight, drugs can be developed strategically [2]. This approach has been used to develop drugs targeting diseases such as male erectile dysfunction, alopecia, stress urinary incontinence, and premature ejaculation.

The value of animal experiments is that they are a painstaking process to find answers to the "central question" of the mechanisms underlying biological phenomena.

\section{REFERENCES}

1. Hussaini SM, Jang MH. New roles for old glue: astrocyte function in synaptic plasticity and neurological disorders. Int Neurourol J 2018;22(Suppl 3):S106-114

2. Ko IG. Applications of Basic Experimental and Clinical Research Using Biomarkers. Int Neurourol J 2018;22:226-7. 